
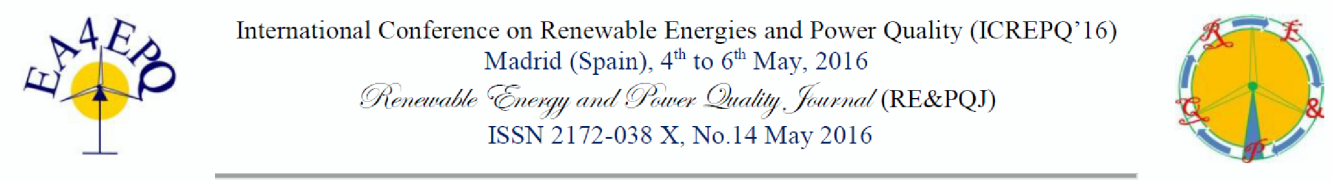

\title{
Supervision of Community Based Microgrids: an Economic Model Predictive Control approach
}

\author{
Francesco Tedesco ${ }^{\star}$, Lubna Mariam ${ }^{\star \star}$, Malabika Basu ${ }^{\star \star}$, Alessandro Casavola ${ }^{\star}$ and Michael F. Conlon ${ }^{\star \star}$ \\ * University of Calabria, DIMES, Via P. Bucci, 42/C - 87036 Rende (CS), Italy. \\ \{casavola, ftedesco\}@dimes.unical.it
}

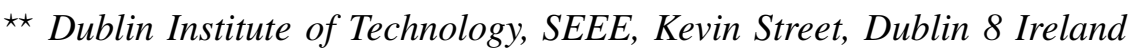
lubna.mariam@gmail.com,mbasu@ieee.org,michael.conlon@dit.ie

\begin{abstract}
In this paper, an Economic Model Predictive Control (EMPC) approach has been presented to manage a Community-based microgrid $(\mathrm{C}-\mu \mathrm{GCC})$ at the pricing level. The main task is at satisfying the demand at prosumer sides and, at the same time, optimizing various $\mu$-Grid contrasting objectives. Emphasis has been given to the operational constraints related to the components lifetime, whose satisfaction would be beneficial for the grid in that the maintenance and replacement costs would be reduced. A simulative analysis has been carried out on the basis of available measured data related to a location in Dublin, Ireland. Results show the effectiveness of implementing the EMPC approach to optimally manage the system.
\end{abstract}

Key words: Renewable Energy, Model Predictive Control, Battery Lifetime, Microgrids Management, Economics of Renewable Energy Systems

\section{INTRODUCTION}

Modern power grids, combining the presence of distributed energy resources (DG) with advanced power electronic and ICT systems, introduce several new concepts and architectures as Microgrid ( $\mu$ Grid). Microgrids usually represent a single organized power subsystem having a number of distributed generation (DG) sources, both renewable and/or conventional, energy storage system (ESS), and a cluster of loads [1]. These systems have been identified as an easy way to integrate micro-generators into the Low-Voltage (LV) grids [2].

Several countries (e.g. Ireland) have some policies for micro generation ( $\mu \mathrm{Gen}$ ) but $\mu$ Grid policies are still missing. In this context, a modified form of Grid, referred to as Community-Based Microgrid (C- $\mu$ Grid), has been proposed in [3], [4]. C- $\mu$ Grid is a system where people in a community having their own $\mu$ Gen (existing or newly purchased) systems decide to cluster and form a $\mu$ Grid system. This paper deals with this new concept of grids and wants to address the scenario depicted in Figure 1. There, a grid connected C$\mu$ Grid system with storage capabilities has been developed from a cluster of $\mu \mathrm{Gen}$ systems. Each of the community users is provided with his/her own micro-wind turbines (as $\mu \mathrm{Gen})$ and, instead of having separate multiple converters, they are all connected through a central converter.

All $\mu$ Gens are connected in parallel to a common DC bus and have their own charge controller (CC). A common

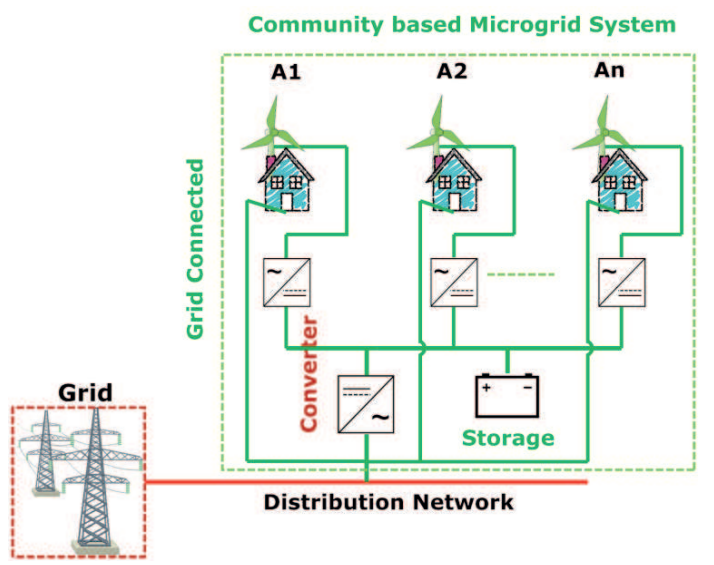

Fig. 1. Grid-connected C- $\mu$ Grid system with storage

storage is integrated to the dc bus. All converters have the same dc output voltage. The dc bus is connected to a central inverter that is managed by a Community Microgrid Central Controller (C- $\mu \mathrm{GCC})$, behaving according to its own control strategy/algorithm, that is in charge of regulating the level of energy to be stored and the amount of energy to be imported from/exported to the main grid in order to satisfy the demands while minimizing the cost of energy (COE) of the system.

In this context, the control strategy adopted by the C$\mu \mathrm{GCC}$ is crucial to facilitate the power flow among the generators, the storage unit and the loads. In this respect, the paper proposes a control algorithm for $\mathrm{C}-\mu \mathrm{GCC}$ to manage the power shared among the prosumers on the basis of the energy demanded at the consumer sides, the energy produced by the $\mu$ Gens and the buying/selling tariff related to the energy exchange with the external main grid. The proposed strategy is based on the emerging Economic Model Predictive Control (Economic MPC) approach.

Economic MPC is a receding horizon control strategy that differs from standard MPC in that its action is computed online by minimizing an objective function that is related to some economical aspects of the system management rather than control objectives, such as stability or tracking perfor- 
mance. Potential of Economic MPC for power management has been investigated in [5], where such a method was used to operate a portfolio of power generators and consumers so that the cost of producing the required power is minimized. Following the same lines, the above problem has been investigated in the presence of massive energy storage facilities in [6] while a more efficient formulation of Economic MPC has been presented in [7] for the minimization of the production costs. Other works focusing on MPC control of energy systems can be found e.g. in [8], [9], [10], [11], [12], [13], [14], [15].

Notice that none of mentioned works consider in an explicit way any constraint related to components lifetime. However, a proper management of the components of the system aimed at alleviating their degradation should lead to benefits in terms of reduction of both maintenance and replacement costs.

Moving from this considerations, in this work, an Economic MPC framework is developed for the optimal realtime power dispatch in a $\mathrm{C}$ - $\mu$ Grid while minimizing the operational costs of the energy system. Differently from existing works on the topic, the proposed strategy comes equipped with the capability of taking into account in an explicit way the lifetime of the battery during the computation of the control commands. In the paper, such a feature is addressed in two different ways by acting, in a first formulation, on the cost index of the optimization problem, whereas, in an alternative scheme, by adding an explicit constraint on the desired lifetime in the optimization problem by exploiting the notion of battery throughput [16].

The simulation results show the effectiveness of the solutions, in terms of COE and components lifetime, in properly distributing the power amongst the prosumers, storage and the grid. Dublin, Ireland, has been chosen as the geographical location for this study.

This paper is organized as follows. In Section II the model of the plant is described and the problem to be solved formulated. The Economic MPC control problem is formulated in Section III. In Section IV, simulations are used to show the effectiveness of the proposed approach.

\section{NOTATIONS}

Let $\mathbb{I}_{\geq 0}$ denote the set of nonnegative integers, $\mathbb{I}_{p: q}, p<q$ the integer sequence $\{p, p+1, p+2, \ldots, q\}$ and $\mathbb{R}$ the set of real numbers.

\section{C- $\mu$ Grid Operational Control Statement}

\section{A. Control-oriented Modelling}

For our purposes, the $\mathrm{C}-\mu \mathrm{Grid}$ system under investigation can be described by the model depicted in Figure 2, where the $\mu$ Grid is represented by the interconnection of four main components which include: a local consumer, a renewable generator (wind turbine), a storage facility (battery) and an external grid (main grid). In the figure, the signals $d_{l}(t)$ (kilowatthaour) and $d_{s}(t), t \in \mathbb{I}_{\geq 0}$ collect all the demand required by consumers and the entire energy produced by the wind sources respectively. Moreover, $u_{b}(t)$ denotes the

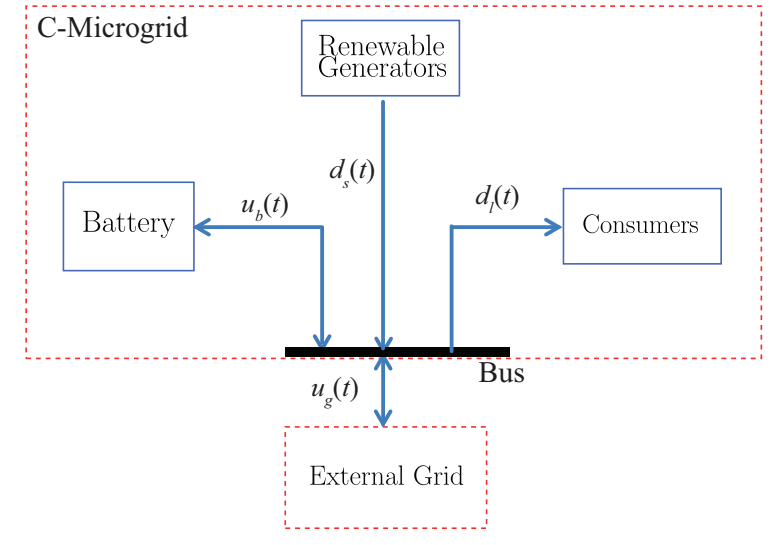

Fig. 2. C- $\mu$ Grid control oriented scheme

energy transmitted/received to/from the battery storing a certain amount of energy $x(t)$, while $u_{g}(t)$ represents either the energy bought from the main grid $\left(u_{g}(t)>0\right)$ or the energy sold to the main grid $\left(u_{g}(t)<0\right)$ within the following fixed bounds

$$
-\underline{u}_{g} \leq u_{g}(t) \leq \bar{u}_{g}
$$

The cost of purchasing power from the grid varies according to the time-varying buying tariff $\alpha(t)>0$ (euros per kilowatthour) while the selling income is regulated by a different time-varying tariff $\beta(t)>0$. In this work, we assume that $\alpha(t) \geq \beta(t), \forall t \in \mathbb{I}_{\geq 0}$.

The interactions among the independent components of the $\mathrm{C}-\mu \mathrm{Grid}$ is allowed by the bus that enable power exchange from the wind turbines and main grid to battery and loads according to the following algebraic equation

$$
d_{l}(t)=-u_{b}(t)+u_{g}(t)+d_{s}(t)
$$

where only the quantities $u_{b}(t)$ and $u_{g}(t)$ are assumed to be directly controllable by the supervisor while $d_{s}(t)$ and $d_{l}(t)$ are stochastic power flows driven by wind turbine sources and the consumer load demands respectively.

The battery is modeled as a device capable of storing a certain amount of dc electricity. Limits are specified on how quickly it can be charged or discharged, how deeply it can be discharged without causing damage and how much energy can cycle through it before it needs replacement. Moreover, it is assumed that the properties of the battery remain constant throughout its lifetime and are not affected by external factors such as temperature.

In the proposed $\mathrm{C}$ - $\mu$ Grid setting, for describing the battery operation (the charge and discharge modes), we use a quasikinetic battery model [17], which models the battery as a tank storing a certain amount of energy $x(t)$ at time step $t$ that evolves according to the following discrete-time difference equation

$$
x(t+1)=\tau x(t)+u_{b}(t)
$$

with $\tau \leq 1$ denoting the hourly self-discharge decay [18]. Obviously, the quantity of storable energy is constrained as the capacity of the battery is limited, i.e.

$$
x(t) \leq \bar{x}
$$


Furthermore, a further constraint about the minimum level of stored energy should be taken into account

$$
x(t) \geq \underline{x}
$$

Moreover, according to the kinetic battery model, only a certain amount of stored energy is immediately available for charging or discharging, but the rest is chemically bound. For this reason the following inequalities are considered

$$
\underline{u}_{b} \leq u_{b}(t) \leq \bar{u}_{b}
$$

in order to limit the amount of transferable energy from/to the battery to/from the other $\mu$ grid components. Finally, in order to include in an explicit way the lifetime of the battery in our supervision scheme, we have equipped the above battery model with the further difference equation

$$
q(t+1)=q(t)-\left|u_{b}(t)\right|
$$

where $q(t)$ is the remaining lifetime throughput of the battery at time $t$, that is the amount of energy that can cycle through the battery before failure, in practice, when $q(t) \approx 0$, the battery should be replaced.

\section{B. System Operational Goals}

Different criteria may be taken into account when managing a $\mathrm{C}-\mu \mathrm{Grid}$. In this paper and according to a given context, the operational goals in the management of the $\mathrm{C}-\mathrm{C}-\mu \mathrm{Grid}$ are of three kinds: economic, safety and durability and are respectively stated as follows:

1) To provide a reliable electricity supply minimizing the electricity purchase from the external grid;

2) To guarantee the availability of enough energy in the battery to satisfy the consumer stochastic demand under the stochastic power flow provided by the wind turbines.

3) To plan an optimized battery schedule that guarantees a long lifetime.

The economic goal could be achieved by minimizing $\alpha(t) u_{g}(t)$ when buying while maximizing $-\beta(t) u_{g}(t)$ when selling. As a consequence, a supervisor for the grid should indeed decide also if $u_{g}(t)$ should be positive or negative. All these requirements could lead to an optimization problem formulation involving both boolean and real variables. In order to avoid a mixed-integer program, we encode the above criteria into a standard optimization problem first by recasting $u_{g}(t)$ as

$$
u_{g}(t)=u_{g}^{+}(t)-u_{g}^{-}(t)
$$

where

$$
\begin{aligned}
& 0 \leq u_{g}^{+}(t) \leq \bar{u}_{g} \\
& 0 \leq u_{g}^{-}(t) \leq \underline{u}_{g}
\end{aligned}
$$

in this way we are splitting the energy exchanged with the grid $u_{g}(t)$ into two virtual flows: the sold energy $u_{g}^{-}(t)$ and the bought energy $u_{g}^{+}(t)$. Secondly, the above formulation allows us to adopt the following performance indicator

$$
J_{E}(t) \triangleq \alpha(t) u_{g}^{+}(t)-\beta(t) u_{g}^{-}(t)
$$

The safety goal could be enforced by the safety constraint (5), which can be conveniently reformulate as a soft constraint in the following way:

$$
x(t) \geq \underline{x}(t)-\xi(t) \geq \mathbf{0} \quad \forall t,
$$

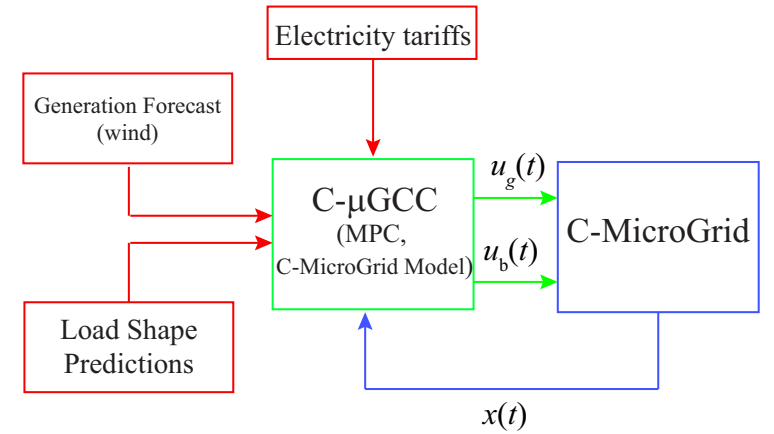

Fig. 3. C- $\mu \mathrm{GCC}$ scheme

where $\underline{x} \in \mathbb{R}$ is a safety threshold of energy, estimated empirically, above which is desired to keep the level of energy to cope with the risk involved in the certaintyequivalence principle due to demand uncertainty and in order to avoid a deep discharge of the battery. As a result, the new performance index

$$
J_{S}(t) \triangleq \xi^{2}(t)
$$

is stated.

Finally, the third goal (durability) can be achieved by considering the following performance index

$$
J_{D}(t) \triangleq u_{b}^{2}(t)
$$

which aims at reducing the charge/discharge operations. In the next section an alternative solution involving equation (7) is presented in order to deal with this third goal.

\section{ECONOMIC MPC FOR C- $\mu$ GRID OPERATIONAL GOAL}

The main goal of the operational control of microgrid at pricing level is to satisfy the demands at consumer sides, and optimizing, at the same time, the management policies expressed as a multi-objective optimal control problem. Hence, MPC results a suitable technique to control a C- $\mu$ Grid because its capability to deal efficiently with multi-variable dynamic constrained systems and predict the proper actions to achieve the optimal performance according to a userdefined cost function. Moreover, the MPC design follows a systematic procedure [19], which generates the control input signals to the plant by combining a prediction model and a receding-horizon control (RHC) strategy.

In this work we introduce two Economic MPC strategies that deal with the economic and safety goals in the same way but adopt different criteria to cope with the durability goal. Both strategies are based on the control scheme depicted in Figure 3 where the $C-\mu G C C$ to be designed makes use of the current state of the battery and wind generation and load demand forecasts. In this paper, however, it is assumed that the supervisor has perfect knowledge of the future evolution of the mentioned quantities.

The first EMPC algorithm we are going to introduce is referred to as $\mathrm{MPC}_{1}$. Consider the system (3) at a measured condition. Given a prediction horizon $H_{p}=48$, and the 
control objectives (see (10) and (12)) aggregated in a performance index $J: \mathbb{R}^{H_{p} \times\left(H_{p}-1\right)} \rightarrow \mathbb{R}$, the $\mathrm{MPC}_{1}$ problem for the microgrid consists in solving a finite horizon optimal control problem given by

$$
J_{1}^{*} \triangleq \min _{u_{g}^{+}, u_{g}^{-}, u_{b}, \xi} \sum_{k=t}^{t+H_{p}-1}\left[\gamma_{E} J_{E}(k \mid t)+\gamma_{S} J_{S}(k \mid t)+\gamma_{D} J_{D}(k \mid t)\right]
$$

subject to:

$$
\begin{aligned}
& x(k+1 \mid t)=\tau x(k \mid t)+u_{b}(k \mid t), \forall k \in \mathbb{I}_{t: t+H_{p}} \\
& d_{l}(k \mid t)=-u_{b}(k \mid t)+u_{g}(k \mid t)+d_{s}(k \mid t) \\
& x(k+1 \mid t) \leq \bar{x}, \forall k \in \mathbb{I}_{t: t+H_{p}} \\
& -\underline{u_{b}} \leq u_{b}(k \mid t) \leq \bar{u}_{b}, \forall k \in \mathbb{I}_{t: t+H_{p}-1} \\
& 0 \leq u_{g}^{+}(k \mid t) \leq \bar{u}_{g}, \forall k \in \mathbb{I}_{t: t+H_{p}-1} \\
& 0 \leq u_{g}^{-}(k \mid t) \leq \underline{u}_{g}, \forall k \in \mathbb{I}_{t: t+H_{p}-1} \\
& x(k+1 \mid t) \geq \underline{x}-\xi(k+1 \mid t) \geq 0, \forall k \in \mathbb{I}_{t: t+H_{p}} \\
& \left(x(t \mid t), d_{l}(t \mid t), d_{s}(t \mid t)\right)=\left(x(t), d_{l}(t), d_{s}(t)\right)
\end{aligned}
$$

Then, according to the RHC strategy, one applies only the first samples $u_{g}^{+}(t \mid t), u_{g}^{-}(t \mid t), u_{b}(t \mid t)$ of the optimal sequences

$$
\begin{array}{rrr}
\vec{u}_{g}^{+}(x(t)) & \triangleq & {\left[u_{g}^{+}(t \mid t), \ldots, u_{g}^{+}\left(t+H_{p}-1 \mid t\right)\right]} \\
u_{g}^{-}(x(t)) \triangleq & \left.\triangleq u_{g}^{-}(t \mid t), \ldots, u_{g}^{-}\left(t+H_{p}-1 \mid t\right)\right] \\
\overleftrightarrow{u}_{b}(x(t)) \triangleq & \triangleq & {\left[u_{b}(t \mid t), \ldots, u_{b}\left(t+H_{p}-1 \mid t\right)\right]}
\end{array}
$$

respectively. At the next time instant, the prediction horizon is shifted one time instant ahead and the optimization is restarted with new feedback measurements and updated predictions to compensate unmeasured disturbances and model inaccuracies. This scheme is repeated at each future time instant.

Please notice that in the above optimization problem the durability goal is enforced by including in the optimization cost the term $J_{D}$ introduced in the previous section. On the contrary, such a goal can be dealt with by including explicit constraints involving the battery lifetime as in the following second MPC formulation denoted as $\mathrm{MPC}_{2}$

$$
J_{2}^{*} \triangleq \min _{u_{g}^{+}, u_{g}^{-}, u_{b}, \xi} \sum_{k=t}^{t+H_{p}-1}\left[\gamma_{E} J_{E}(k \mid t)+\gamma_{S} J_{S}(k \mid t)\right],
$$

subject to:

$$
\begin{aligned}
& (14 b)-(14 i) \\
& q(k+1 \mid t)=q(k \mid t)-\left|u_{b}(k \mid t)\right|, \forall k \in \mathbb{I}_{t: t+H_{p}-1} \\
& \frac{D(t)}{2} \sum_{k=t}^{t+H_{p}-1}\left(\left|u_{b}(k \mid t)\right|\right) \leq q(t) .
\end{aligned}
$$

where the quantity $D(t)$ is the desired remaining amount of days at time $t$ before battery needs replacement. Roughly speaking, the above solution is computed in such a way that if the same quantity of energy $\sum_{k=t}^{t+H_{p}-1}\left(\left|u_{b}(k \mid t)\right|\right)$ were transferred to/from the battery from time $t$ onward, then the battery would have a lifetime at least equal to $D(t)$. Even in this case the RHC approach applies and, furthermore, the quantity $D(t)$ should be decreased by 1 at each time $t$ instant, i.e. $D(t+1)=D(t)-1$.

Remark 1: Despite the intuitive formulation of the RHC strategy, the on-line tuning of an MPC controller is not trivial or systematic. The MPC tuning parameters for the given cost function usually are the prediction horizon $H_{p}$ and the weighting terms $\gamma_{E}, \gamma_{S}, \gamma_{D}$.

Remark 2: It is worth commenting that in problem (17) $u_{b}(k \mid t) \rightarrow 0$ when $D(t) \rightarrow \infty$, that is if the desired remaining amount of days before replacement for the battery is too high, its activity results very limited or even inexistent.

\section{Application to AN IRISh COMMUNity C- $\mu$ GRID}

\section{A. Case Study Description}

In this section the system depicted in Figure 1 is set in the Irish context. In this respect, specific Grid electricity bill for day time and night time (see Table 1) are taken from the local authority of electricity, $\mathrm{ESB}^{1}$. Details about system parameters and economical information can be found in Table 1. Here it is worth commenting that the decay $\tau$ is tuned such that the battery loses the $20 \%$ of the stored energy after one month.

TABLE I

SYSTEM PARAMETERS AND COSTS

\begin{tabular}{lc}
\hline HOUSES & \\
\hline NUMBER & 10 \\
AVERAGE DAILY SINGLE LOAD DEMAND & $14 \mathrm{kWh} / \mathrm{day}$, \\
\hline TURBINES & \\
\hline MAXIMUM ACHIEVABLE POWER & $6 \mathrm{~kW}$ \\
COST PER UNIT & $18 \mathrm{k} €$ \\
\hline BATTERY & \\
\hline CAPACITY $\bar{x}$ & $144 \mathrm{kWh}$ \\
DECAY FACTOR $\tau$ & 0.9997 \\
INITIAL LIFETIME THROUGHPUT Q $(0)$ & $2.5 \times 10^{5} \mathrm{kWh}$ \\
MINIMUM STORABLE ENERGY $\underline{x}^{-}$ & $0.3 \bar{x}$ \\
MAXIMUM CHARGE RATE $\bar{u}_{b}$ & $5 \mathrm{kWh}$ \\
MINIMUM CHARGE RATE $\underline{u}_{b}$ & $5 \mathrm{kWh}$ \\
REPLACEMENT COST & $18 \mathrm{k} €$ \\
\hline CONVERTER & \\
\hline NUMBER & $13430 €$ \\
INITIAL COST & $7597 €$ \\
REPLACEMENT COST & \\
\hline OTHER PARAMETERS & $0.233 € / \mathrm{kWh}$ \\
\hline DAILY BUYING TARIFF $\alpha(t)$ & $0.153 € / \mathrm{kWh}$ \\
NIGHTLY BUYING TARIFF $\alpha(t)$ & $0.103 € / \mathrm{kWh}$ \\
DAILY SELLING TARIFF $\beta(t)$ & $0.103 € / \mathrm{kWh}$ \\
NIGHTLY SELLING TARIFF $\beta(t)$ & $10 \mathrm{kWh}$ \\
MAXIMUM PURCHASABLE ENERGY $\bar{u}_{g}$ & $40 \mathrm{kWh}$ \\
MAXIMUM SALABLE ENERGY $\underline{u}_{g}$ & \\
GRID & $0.233 € / \mathrm{kWh}$ \\
\hline DAILY BUYING TARIFF $\alpha(t)$ & $0.153 € / \mathrm{kWh}$ \\
NIGHTLY BUYING TARIFF $\alpha(t)$ & $0.103 € / \mathrm{kWh}$ \\
DAILY SELLING TARIFF $\beta(t)$ & $10 \mathrm{kWh}$ \\
NIGHTLY SELLING TARIFF $\beta(t)$ & $40 \mathrm{kWh}$ \\
MAXIMUM PURCHASABLE ENERGY $\bar{u}_{g}$ & \\
MAXIMUM SALABLE ENERGY $\underline{u}_{g}$ & \\
\hline OTHER PARAMETERS & \\
\hline MAINTENANCE COST & \\
\hline
\end{tabular}

${ }^{1}$ Electric Ireland, ESB; https://www.electricireland.ie/ei/home/index.jsp 


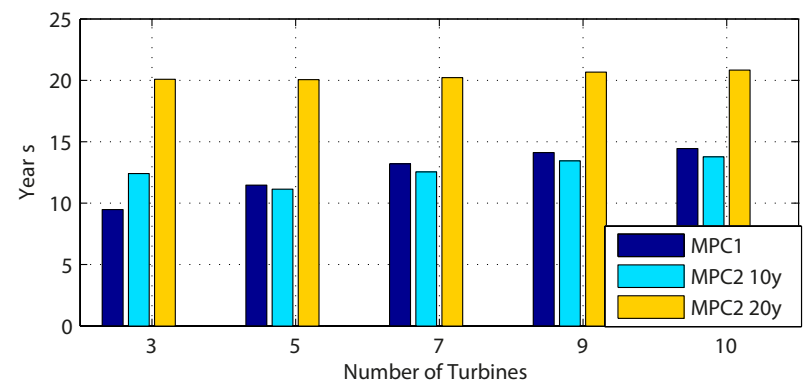

Fig. 4. Expected battery lifetime

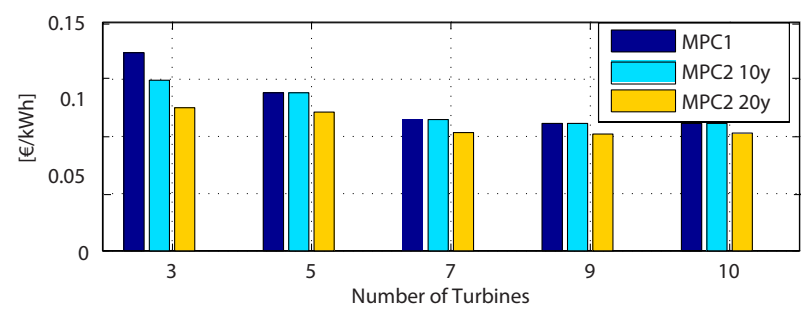

Fig. 5. Cost of energy (COE)

\section{B. Closed loop setup}

All results have been obtained by considering an one year real-demand data (sampled at 1 hour interval), and $H_{p}=$ 48. For all MPC strategies, the control objectives in (14a) have been prioritized with $\gamma_{E}=1, \gamma_{S}=0.001$ and $\gamma_{D}=$ 0.0001 , which resulted suitable after a trial-and-error tuning strategy. The network has been simulated by using the same model used to design the MPC controller but fed with real energy demands. Wind speed data has been collected from MET Éireann ${ }^{2}$ and load demand for general household in Dublin have been collected from the respective authorities ${ }^{3}$. All simulations have been undertaken by using the Yalmip interpreter [20] and the CPLEX solver, all running under MATLAB ${ }^{\complement} 8.2$ environment, running on an Intel ${ }^{\complement}$ Core i5-3330 machine with $3.3 \mathrm{GHz}$ and $8 \mathrm{~GB}$ RAM.

\section{Results, Comparisons and Discussion}

Simulations have been carried out where an increasing number of turbines has been considered in order to test the robustness of the following algorithm:

${ }^{2}$ Monthly wind speed data, Met Éireann; 2012

${ }^{3}$ Standard load profiles, 2011, Retail Market Design Service; 2009

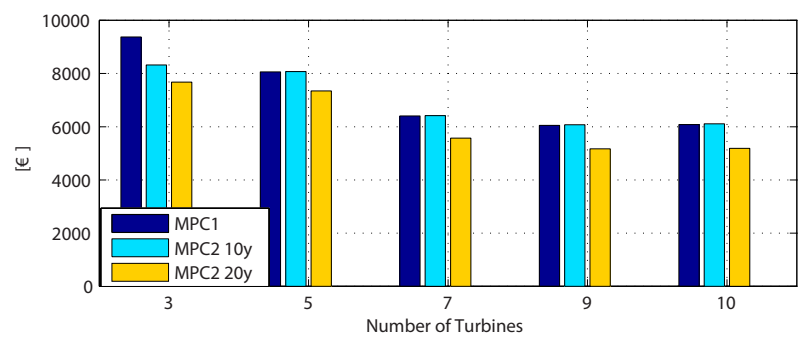

Fig. 6. Projection of neat total costs (system costs minus incomes) over 20 years

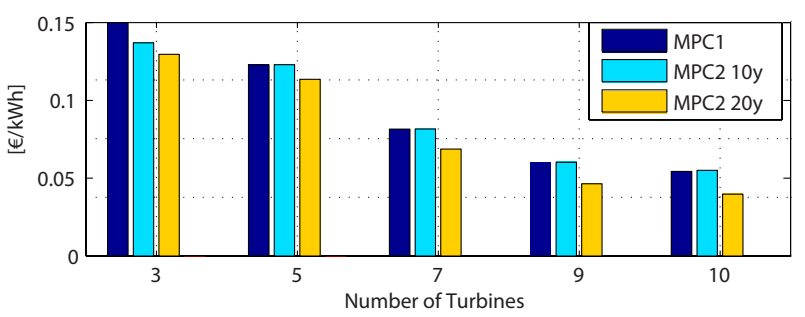

Fig. 7. Cost of energy from $\mathrm{C}-\mu \mathrm{Grid}$ perspective $(\mu \mathrm{COE})$ that is equivalent to the price of demanded kilowatthour for the consumers.

- $\mathrm{MPC}_{1}$ : solving problem (16)

- $\mathrm{MPC}_{2}-10 \mathrm{y}$ : solving problem (17) with desired lifetime for the battery equal to 10 years $(D(0)=10 \times 365)$

- $\mathrm{MPC}_{2}$-20y: solving problem (17) with desired lifetime for the battery equal to 20 years $(D(0)=20 \times 365)$

In Figure 4 the time before battery replacement (i.e the time before $q(t) \approx 0$ ) is depicted. Interesting enough, only the $\mathrm{MPC}_{2}-20 \mathrm{y}$ strategy is able to guarantee a 20 years lifetime for the battery, thus avoiding its replacement while keeping similar performance with respect to its competitors. Such an aspect has a positive impact on the overall operational costs of the C- $\mu$ Grid over an horizon of 20 years (see Figures 4-7). In this respect all calculations are based on a loan having a $4.75 \%$ rate An annual inflation rate of $1.79 \%$ is also taken into account, which was the average annual inflation rate for Ireland between January 2003 and December $2013^{4}$.

Figure 5 analyzes the economic impact of the $\mathrm{C}-\mu \mathrm{Grid}$ from the External Grid point of view by showing the Cost of Energy (COE) that indicates how much is the energy produced by the $\mathrm{C}-\mu \mathrm{Grid}$ (sold energy plus served energy to the load):in this case the $\mathrm{C}-\mu \mathrm{Grid}$ can be seen as a generator.

In order to analyze the economic impact with respect to $\mathrm{C}-\mu$ Grid point of view we have shown the total costs arising from $\mathrm{C}-\mu \mathrm{Grid}$ management, including the incomes derived from the energy export as a negative cost, in Figure 6. Incomes are computed as

$$
\sum_{t=0}^{365 \times 24} \begin{cases}-\alpha(t) u_{g}(t), & u_{g}(t) \geq 0 \\ -\beta(t) u_{g}(t), & u_{g}(t)<0\end{cases}
$$

Such costs have been used to compute the cost of demanded load energy in the $\mathrm{C}-\mu \mathrm{Grid}(\mu \mathrm{COE})$, which represents the price of a kilowatthour for the consumers in the $\mathrm{C}-\mu \mathrm{Grid}$. It is evident, from the above described Figures that the $\mathrm{C}-\mu \mathrm{Grid}$ presents better economic performance with the maximum number of turbines. Moreover, it is worth pointing out that in the case of $\mathrm{MPC}_{2}-20 \mathrm{y}$ both the $\mathrm{COE}$ and the $\mu \mathrm{COE}$ are reduced by $25 \%$ with respect to other presented MPC based approaches. Hence, the proposed strategy that takes into account in an explicit way the battery degradation has not a marginal impact on the economic aspects.

For the sake of completeness we included in Figures 8-9 the time-domain plots pertaining to the first week of

${ }^{4}$ http://www.inflation.eu/inflation-rates/ireland/historic-inflation/cpiinflation-ireland-2014.aspx 

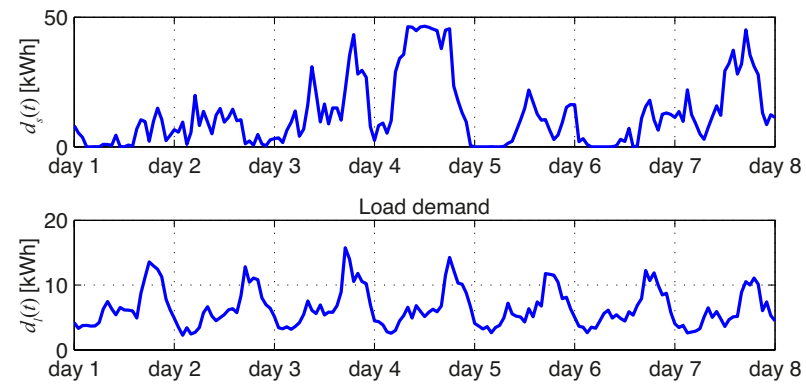

Fig. 8. Up: generated energy; Bottom: Load demand
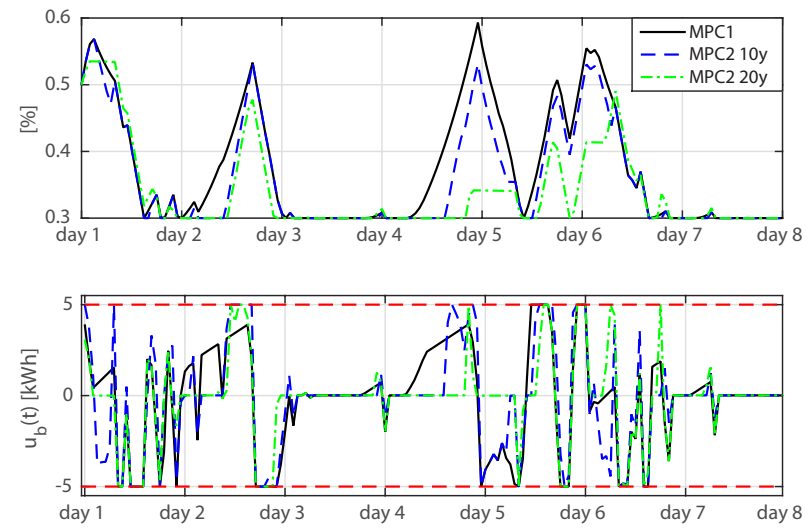

Fig. 9. Up: State of Charge (SOC); Bottom: Energy transferred to/from battery

the simulation horizon. There, the Economic MPC based strategies have been compared in the case of 7 turbines only being operating. It is evident that in Figure 9 both $\mathrm{MPC}_{1}$ and $\mathrm{MPC}_{2}-10 \mathrm{y}$ make a deeper usage of the battery.

\section{Conclusions}

C- $\mu$ Grid can become a transitional solution in countries where policies for $\mu$ Gens are present while those for $\mu \mathrm{Grid}$ don't yet exist. An Economic MPC (EMPC) approach has been applied here to design the central controller of a $\mathrm{C}$ $\mu$ Grid system and shown that has the capability to deal efficiently with multi-variable dynamic constrained systems and predict properly its actions in order to achieve the optimal performance according to user defined cost functions.

A simulative analysis undertaken in a location in Ireland has shown that the control actions provided by the MPC approach were able to feasibly operate the $\mathrm{C}$ - $\mu$ Grid also for a lower number of wind turbines (three in the examples considered). The Economic MPC approach was shown to be able to guarantee a 20 years lifetime for the battery avoiding its replacement while satisfying the other required prescriptions. In particular, it has been shown that the control strategy may have a strong impact on the overall cost of the system, having the MPC approach reduced the COE remarkably. Finally, because all simulations have been performed on hourly basis, the results also show that the MPC approach satisfies the hourly demand. Thus, the approach has the potential to become of industrial interest.

\section{REFERENCES}

[1] R. H. Lasseter and P. Paigi, "Microgrid: a conceptual solution," in Power Electronics Specialists Conference, 2004. PESC 04. 2004 IEEE 35th Annual, vol. 6. IEEE, 2004, pp. 4285-4290.

[2] D. Pudjianto, G. Strbac, F. van Oberbeeke, A. Androutsos, Z. Larrabe, and J. T. Saraiva, "Investigation of regulatory, commercial, economic and environmental issues in microgrids," in 2005 International Conference on Future Power Systems. IEEE, 2005, pp. 6-9.

[3] L. Mariam, M. Basu, and M. F. Conlon, "Community microgrid based on micro-wind generation system," in Power Engineering Conference (UPEC), 2013 48th International Universities'. IEEE, 2013, pp. 1-6.

[4] L. Mariam, M. Basu, and M. F. Conlon, "Sustainability of gridconnected community microgrid based on micro-wind generation system with storage," in 2014 IEEE 23rd International Symposium on Industrial Electronics (ISIE). IEEE, 2014, pp. 2395-2400.

[5] T. G. Hovgaard, K. Edlund, and J. Bagterp Jorgensen, "The potential of economic mpc for power management," in 2010 49th IEEE Conference on Decision and Control (CDC). IEEE, 2010, pp. 7533-7538.

[6] O. Adeodu and D. J. Chmielewski, "Control of electric power transmission networks with massive energy storage using economic mpc," in American Control Conference (ACC), 2013. IEEE, 2013, pp. 58395844.

[7] L. Standardi, N. K. Poulsen, J. B. Jorgensen, and L. E. Sokoler, "Computational efficiency of economic mpc for power systems operation," in Innovative Smart Grid Technologies Europe (ISGT EUROPE), 2013 4th IEEE/PES. IEEE, 2013, pp. 1-5.

[8] W. Qi, J. Liu, X. Chen, and P. D. Christofides, "Supervisory predictive control of standalone wind/solar energy generation systems," Control Systems Technology, IEEE Transactions on, vol. 19, no. 1, pp. 199207, 2011.

[9] A. Parisio and L. Glielmo, "Energy efficient microgrid management using model predictive control," in 2011 50th IEEE Conference on Decision and Control and European Control Conference (CDC-ECC). IEEE, 2011, pp. 5449-5454.

[10] X. Wang, A. Palazoglu, and N. H. El-Farra, "Operation of residential hybrid renewable energy systems: Integrating forecasting, optimization and demand response," in American Control Conference (ACC), 2014. IEEE, 2014, pp. 5043-5048.

[11] L. Xie and M. D. Ilic, "Model predictive economic/environmental dispatch of power systems with intermittent resources," in Power \& Energy Society General Meeting, 2009. PES'09. IEEE. IEEE, 2009, pp. 1-6.

[12] E. Biyik and R. Chandra, "Optimal control of microgrids-algorithms and field implementation," in American Control Conference (ACC), 2014. IEEE, 2014, pp. 5003-5009.

[13] I. Prodan and E. Zio, "A model predictive control framework for reliable microgrid energy management," International Journal of Electrical Power \& Energy Systems, vol. 61, pp. 399-409, 2014.

[14] A. Hooshmand, B. Asghari, and R. Sharma, "Efficiency-driven control of dispatchable sources and storage units in hybrid energy systems," in American Control Conference (ACC), 2014. IEEE, 2014, pp. 16861691.

[15] R. Palma-Behnke, C. Benavides, F. Lanas, B. Severino, L. Reyes, J. Llanos, and D. Sáez, "A microgrid energy management system based on the rolling horizon strategy." IEEE Transactions on Smart Grid, vol. 4, no. 2, pp. 996-1006, 2013.

[16] A. Andersson, "Battery lifetime modelling," Riso National Laboratory, vol. 3, p. 4, 2006.

[17] J. F. Manwell and J. G. McGowan, "Lead acid battery storage model for hybrid energy systems," Solar Energy, vol. 50, no. 5, pp. 399-405, 1993.

[18] A. McEvoy, T. Markvart, L. Castañer, T. Markvart, and L. Castaner, Practical handbook of photovoltaics: fundamentals and applications. Elsevier, 2003.

[19] J. Maciejowski, Predictive control with constraints. Essex, England: Prentice Hall, 2002.

[20] J. Löfberg, "Yalmip : A toolbox for modeling and optimization in MATLAB," in Proceedings of the CACSD Conference, Taipei, Taiwan, 2004. [Online]. Available: http://users.isy.liu.se/johanl/yalmip 